\title{
Deep-learning convolutional neural networks with transfer learning accurately classify COVID19 lung infection on portable chest radiographs
}

\author{
Shreeja Kikkisetti ${ }^{1}$, Jocelyn Zhu ${ }^{1}$, Beiyi Shen ${ }^{2}$, Haifang Li $^{2}$, Tim Duong ${ }^{\text {Corresp. } 1}$ \\ ${ }^{1}$ Radiology, Montefiore Medical Center and Albert Einstein College of Medicine, Bronx, New York, United States \\ 2 Radiology, State University of New York at Stony Brook, Stony Brook, New York, United States \\ Corresponding Author: Tim Duong \\ Email address: tim.duong@stonybrook.edu
}

Portable chest x-ray ( $\mathrm{CCXR}$ ) has become an indispensable tool in the management of Coronavirus Disease 2019 (COVID-19) lung infection. This study employed deep-learning convolutional neural networks to classify COVID-19 lung infections on PCXR from normal and related lung infections to potentially enable more timely and accurate diagnosis. This retrospect study employed deep-learning convolutional neural network (CNN) with transfer learning to classify based on pCXRs COVID-19 pneumonia ( $\mathrm{N}=455)$ on pCXR from normal $(N=532)$, bacterial pneumonia $(N=492)$, and non-COVID viral pneumonia $(N=552)$. The data was randomly split into $75 \%$ training and $25 \%$ testing, randomly. A five-fold crossvalidation was used for the testing set separately. Performance was evaluated using receiver-operating curve analysis. Comparison was made with CNN operated on the whole pCXR and segmented lungs. CNN accurately classified COVID-19 pCXR from those of normal, bacterial pneumonia, and non-COVID-19 viral pneumonia patients in a multiclass model. The overall sensitivity, specificity, accuracy, and AUC were 0.79, 0.93, and 0.79, 0.85 respectively (whole pCXR), and were $0.91,0.93,0.88$, and 0.89 (CXR of segmented lung). The performance was generally better using segmented lungs. Heatmaps showed that CNN accurately localized areas of hazy appearance, ground glass opacity and/or consolidation on the pCXR. Deep-learning convolutional neural network with transfer learning accurately classifies COVID-19 on portable chest x-ray against normal, bacterial pneumonia or non-COVID viral pneumonia. This approach has the potential to help radiologists and frontline physicians by providing more timely and accurate diagnosis. 


\section{Deep-learning convolutional neural networks with transfer learning}

2

\section{accurately classify COVID19 lung infection on portable chest}

\section{radiographs}

\author{
Shreeja Kikkisetti ${ }^{1}$, Jocelyn Zhu ${ }^{1}$, Beiyi Shen ${ }^{2}$, Haifang Li$^{2}$, Tim Q Duong ${ }^{1}$ \\ ${ }^{1}$ Radiology, Montefiore Medical Center and Albert Einstein College of Medicine, Bronx, \\ New York, United States \\ ${ }^{2}$ Radiology, State University of New York at Stony Brook, Stony Brook, New York, United \\ States
}

Correspondence: Tim Duong, Ph.D., Radiology, Montefiore Medical Center and Albert Einstein College of Medicine, 111 E 210th St, Bronx, NY 10467 Tim.duong@einsteinmed.org, Tel: 718 9206268

Funding: none

Manuscript Type: Original Investigation
Abbreviations
COVID-19
Coronavirus Disease 2019
CXR
chest x-ray
$\mathrm{CNN}$
convolutional neural networks
AI
artificial intelligence 
none

27 Word Count for Text: 2700

\section{ABSTRACT}

Portable chest $\mathrm{x}$-ray (pCXR) has become an indispensable tool in the management of Coronavirus

Disease 2019 (COVID-19) lung infection. This study employed deep-learning convolutional neural networks to classify COVID-19 lung infections on pCXR from normal and related lung infections to potentially enable more timely and accurate diagnosis. This retrospect study employed deep-learning convolutional neural network (CNN) with transfer learning to classify based on pCXRs COVID-19 pneumonia $(\mathrm{N}=455)$ on pCXR from normal $(\mathrm{N}=532)$, bacterial pneumonia $(\mathrm{N}=492)$, and non-COVID viral pneumonia $(\mathrm{N}=552)$. The data was randomly split into $75 \%$ training and $25 \%$ testing, randomly. A five-fold cross-validation was used for the testing set separately. Performance was evaluated using receiver-operating curve analysis. Comparison was made with CNN operated on the whole pCXR and segmented lungs. CNN accurately classified COVID-19 pCXR from those of normal, bacterial pneumonia, and non-COVID-19 viral 41 pneumonia patients in a multiclass model. The overall sensitivity, specificity, accuracy, and AUC 42 were $0.79,0.93$, and $0.79,0.85$ respectively (whole pCXR), and were $0.91,0.93,0.88$, and 0.89 43 (CXR of segmented lung). The performance was generally better using segmented lungs.

44 Heatmaps showed that CNN accurately localized areas of hazy appearance, ground glass opacity 45 and/or consolidation on the pCXR. Deep-learning convolutional neural network with transfer 46 learning accurately classifies COVID-19 on portable chest x-ray against normal, bacterial 
47 pneumonia or non-COVID viral pneumonia. This approach has the potential to help radiologists

48 and frontline physicians by providing more timely and accurate diagnosis.

Key words:

coronavirus; machine learning; chest X-ray; computed tomography; lung infection

\section{Introduction}

Coronavirus Disease 2019 (COVID-19) is a highly infectious disease that causes severe

respiratory illness (Hui et al. 2020; Lu et al. 2020). It was first reported in Wuhan, China in

December 2019 ( $\mathrm{Li}$ et al. 2020c) and was declared a pandemic on Mar 11, 2020 . The first confirmed case of coronavirus disease 2019 (COVID-19) in the United States was reported from

Washington State on January 31, 2020 (Holshue et al. 2020). Soon after, Washington, California and New York reported outbreaks. COVID-19 has already infected 10 million, killed more than 0.5 million people, and the United States has become the worst-affected country, with more than $28,2020)$. There are recent spikes of COVID-19 infection cases across many states and around the world and there will likely be second waves and recurrence.

A definitive test of COVID-19 infection is the reverse transcription polymerase chain reaction (RT-PCR) of a nasopharyngeal or oropharyngeal swab specimen (Tang et al. 2020; Wang et al. 2020). Although RT-PCR has high specificity, it has low sensitivity, high false negative rate,

67 and long turn-around time (Tang et al. 2020; Wang et al. 2020) (currently $\sim 4$ days although 68 improvement and other tests are becoming available (CDC). By contrast, portable chest X-rays 69 (pCXR) is convenient to perform, has a fast turnaround, and is well suited for imaging contagious 
70 patients and longitudinal monitoring of critically ill patients in the intensive care units because the

71 equipment can be readily disinfected, preventing cross-infection. pCXR of COVID-19 infection

72 has certain unique characteristics, such as predominance of bilateral, peripheral, and low lobes

73 involvement, with ground-glass opacities with or without airspace consolidations as the disease

74 progresses. These characteristics generally differ from other lung pathologies, such as bacterial

75 pneumonia or other viral (non-COVID-19) lung infection. Based on CXR and laboratory findings,

76 clinicians might start patients on empirical treatment before the RT-PCR results become available

77 or even if the RT-PCR come back negative due to high false negative rate of RT-PCR. Early

78 treatment in COVID-19 patients is associated with better clinical outcomes. Similarly, computed

79 tomography (CT), which offers relatively more detailed features (such as subtle ground-glass

80 opacity (Li et al. 2020b; Xu et al. 2020)), has also been used in the context of COVID-19. However,

81 CT suite and equipment are more challenging to disinfect, and thus it is much less suitable for

82 examining patients suspected of or confirmed with contagious diseases in general and COVID-19

83 in particular. Longitudinal CT monitoring of critically ill patients in the intensive care units is also

84 challenging. In short, pCXR has become an indispensable imaging tool in the management of

85 COVID-19 infection, is often one of the first examinations a patient suspected of COVID-19

86 infection receives in the emergency room, and ideally used for longitudinal monitoring of critically

87 ill patients in the intensive care units.

88 The usage of pCXR under the COVID-19 pandemic circumstances is unusual in many

89 aspects. For instance, pCXR is preferred as it can be used at the bedside without moving the

90 patients, but the imaging quality is not as good as conventional CXR. In addition, COVID-19

91 patients may not be able to take full inspirations during the examination, obscuring possible

92 pathology, especially in the lower lung fields. Many sicker patients may be positioned on the side 
93 which compromises imaging quality. Thus, pCXR data under the COVID-19 pandemic

94 circumstances are suboptimal and, thus, may be more challenging to interpret. Moreover, pCXR

95 is increasingly read by non-chest radiologists in some hospitals due to increasing demands,

96 resulting in reduced accuracy and efficiency.

97 pCXR images contain important clinical features that could be easily missed by the naked

98 eyes. Computer-aided methods can improve efficiency and accuracy of pCXR interpretations,

99 which in turn provides more timely and relevant information to frontline physicians. Deep-learning

100 artificial intelligence (AI) is increasingly used to analyze diagnostic images (Ehteshami Bejnordi

101 et al. 2018; LeCun et al. 2015). AI has the potential to facilitate disease diagnosis, staging of

102 disease severity and longitudinal monitoring of disease progression.

103 One common machine-learning algorithm is the convolutional neural network (CNN)

104 (Krizhevsky et al. 2012), which takes an input image, learns important features in the image such

105 as size or intensity, and saves these parameters as weights and bias to differentiate types of images

106 (Song et al. 2020). CNN architecture is ideally suited for analyzing images. Moreover, many of

107 the machine learning algorithms are trained to solve specific tasks, where models need to be rebuilt

108 from scratch if the feature changes. Transfer learning overcomes such drawback by utilizing

109 knowledge acquired for one task to solve related ones. Transfer learning is useful when dealing

110 with small sample size data because the pre-trained weights improve efficiency and performance

111 (Byra et al. 2018; Samala et al. 2017).

112 Many artificial intelligence (AI) algorithms based on deep-learning convolutional neural

113 networks have been deployed for pCXR applications (Harris et al. 2019; Heo et al. 2019; Mekov

114 et al. 2020) and these algorithms can be readily repurposed for COVID-19 pandemic

115 circumstances. While there are already many papers describing prevalence and radiographic 
116 features on pCXR of COVID-19 lung infection (see reviews (Bao et al. 2020)), there is a few AI

117 papers (Apostolopoulos \& Mpesiana 2020; Cohen et al. 2020a; Elaziz et al. 2020; Hurt et al. 2020;

118 Murphy et al. 2020; Ozturk et al. 2020; Pereira et al. 2020; Zhu et al. 2020a) to classify CXRs of

119 COVID-19 patients from CXR of normals or related lung infections. The full potential of AI 120 applications of $\mathrm{pCXR}$ under COVID-19 pandemic circumstances is not yet fully realized.

121 The goal of this pilot study is to employ deep-learning convolutional neural networks to 122 classify normal, bacterial infection, and non-COVID-19 viral infection (such as influenza) against 123 COVID-19 infection on pCXR. The performance was evaluated using receiver-operating curve 124 (ROC) analysis. Heatmaps were also generated to visualize and assessment the performance of the 125 AI algorithm.

\section{Materials and Methods}

129 Data sources: This retrospective study used publicly available pCXR of i) COVID-19 infection,

130 ii) non-COVID-19 viral infection, iii) bacterial pneumonia, and iv) normal subjects. The COVID-

13119 pCXR were downloaded from on May 27th, 2020 (Cohen et al. 2020b). The original download 132 contained $673 \mathrm{CT}$ or pCXR images of COVID-19, SARS, acute respiratory distress syndromes, 133 pneumocystis, streptococcus, legionella, Chlamydophila, E Coli, Klebsiella, lipoid, Varicella, and 134 influenza. The labels for the data came from a metadata file associated with the open dataset. The

135 final sample size for COVID-19 patients was 455 pCXR from 197 patients. We recognized that 136 this dataset was a public, community-driven dataset and there are potential selection biases. A 137 radiologist (B.S.) evaluated all images for quality and relevance and each case was COVID-19 
138 positive based on available data. As a result of this evaluation, a few images that were deemed to

139 be of poor quality, were excluded.

140 The other datasets were taken from the established Kaggle chest X-ray image (pneumonia)

141 dataset (https://www.kaggle.com/paultimothymooney/chest-xray-pneumonia). Although the

142 Kaggle database has a large sample size, we randomly selected a sample size comparable to that

143 of COVID-19 to avoid asymmetric sample size bias that could skew sensitivity and specificity.

144 The sample sizes chosen for bacterial pneumonia, non-COVID-19 viral pneumonia, and normal

145 pCXR were 492, 552 and 532 patients, respectively. Similarly, a chest radiologist evaluated all

146 images for quality.

147

148

$C N N$ : A CNN, a type of neural network, is ideally suited for analyzing images. In a standard

149 CNN model, a filter (window) travels over each region of an image and looks for different features

150 such as edges, colorations, patches, and more in order to classify an image into a certain category.

151 Our CNN architecture was based on VGG16 (Figure 1), a convolutional neural network

152 (Simonyan \& A. 2014), architecture was utilized for computation efficiency and ease to

153 implement, for immediate translation potential. Our VGG16 architecture had 13 convolution layers

154 that each run a series of filters over the image to extract important features. The VGG16 model

155 was used because it was pretrained on the ImageNet database and properly employs transfer

156 learning which makes the training process efficient. In other words, instead of having to learn all

157 the relationships in an image from scratch, the model is already familiar with that when transfer

158 learning is employed. The data was normalized first by transforming all files into RGB images and

159 resizing them into $224 \times 224$ pixels to make them compatible with the VGG16 framework. Next,

160 the images were one-hot-encoded and randomly split into $75 \%$ training and $25 \%$ testing. One hot 
161 encoding means to turn all the categorical labels into numerical values containing zeroes and ones

162 to make it much easier for the computer to read. VGG16 implements 13 convolutional layers: 5

163 Max Pooling layers and 3 Dense layers which sum up to 21 layers and 16 weight layers (Ren et al.

164 2020). Conv 1 has 64 filters while Conv 2 has 128 filters, Conv 3 has 256 filters while Conv 4 and

165 Conv 5 have 512 filters. The first two layers have 2 sublayers while the 4 th and 5 th layers have 3

166 sublayers. A sublayer is another layer within a convolutional layer that further filters images and

167 passes information down to the next sublayers. The information collected from all the sublayers is

168 compiled and sent to the next layer to make a cohesive prediction. A max-pooling layer was used

169 after each step in the model to down sample the input and identify its important features based on

170 the methods described in (Ren et al. 2020). A max-pooling layer reduces the dimensionality of the

171 image and allows for assumptions to be made about features contained in the sub regions (Ren et

172 al. 2020). All convolutional layers used rectified linear units (ReLUs) as an activation function

173 because it adds a small number of learnable parameters (Ren et al. 2020). Three fully connected

174 layers were used, each having 4096 nodes. Fully connected layers compose some of the last few

175 layers in a model and connect all the inputs from each layer to the activation unit of the next layer

176 (Ren et al. 2020). Dropout layers were used, along with the Softmax function, to prevent

177 overfitting. Dropout layers work by randomly setting the edges of hidden neurons to 0 at each

178 update of the training phase. The softmax function turns all the scores from the images into a

179 normalized probability distribution, which helps make the final prediction (Ren et al. 2020). For

180 data analysis, batch sizes of 32 were used to limit computational expense and trained for 50 epochs.

181 Epochs can be thought of as iterations. Several optimizers were tested and Adams optimization

182 function was found to yield the lowest validation loss. The learning rate was lowered from the

183 recommended 0.01 to 0.001 to prevent overshooting the global minimum loss. Categorical cross 
184 entropy was used as a loss function since the loss value decreases as the predicted probability

185 converges to the actual label.

186

187

CNN analysis was performed on the whole pCXR as well as virtually segmented lungs.

Lung segmentation was performed using a CNN architecture with 22 convolutional layers, 4 maxpooling layers, and 4 merged layers for connectivity. A ReLu activation function was used with the Keras library. The output consisted of a mask of the segmented lungs. The segmented lungs were then fed into the CNN model for the Covid19 classification. This model was trained on the Montgomery dataset and achieved an IoU score of 0.956 and dice score of 0.972 . one specific output class to obtain the class activation maps, indicating the discriminative image regions $\mathrm{CNN}$ paid attention to.

the test set separately. Performance of the prediction model used standard ROC analysis of the 202 area under the curve (AUC), accuracy, sensitivity, specificity, precision, recall and F1 scores. Precision was computed using true positives divided by the sum of false positives and true positives; Recall was computed using the true positives divided by the sum of true positives and false negatives; F1 scores were the mean of recall and precision rates. 


\section{Results}

208 Figure 2 shows examples of pCXR from a normal subject, patients with bacterial 209 pneumonia, non-COVID-19 viral pneumonia, and COVID-19 infection. COVID-19 is often

210 characterized by ground-glass opacities with or without nodular consolidation with predominance

211 of bilateral, peripheral and lower lobes involvement. Non-COVID-19 viral pneumonia is often

212 characterized by diffuse interstitial opacities, usually bilaterally. Bacterial pneumonia is often

213 characterized by confluent areas of focal airspace consolidation.

214 Figure 3 shows the training and validation loss and accuracy as a function of the epoch of

215 the CNN models. Loss decreases and accuracy improved with increasing epoch for both training 216 and validation dataset. The accuracy typically reached $>0.8$.

217 CNN was used to classify COVID-19 pCXR from those of normal, bacterial pneumonia, 218 and non COVID-19 viral pneumonia patients in a multi-class neural network model. The results 219 of the multi-class CNN classification for the whole CXR in the form of the confusion matrix is 220 shown in Table 1. The precision, recall, and F1 scores for the whole pCXR (Table 2) showed 221 good to excellent performance. For CNN with transfer learning performed on the whole pCXR, 222 the overall sensitivity, specificity, accuracy, and AUC were $0.79,0.93$, and $0.79, .84$ respectively. 223 For CNN performed on segmented lungs, the overall sensitivity, specificity, accuracy, and AUC 224 were $0.91,0.93,0.88,0.89$ respectively. The performance was generally better using segmented 225 lungs.

To visualize the spatial location on the images that the CNN networks were paying attention to for classification, heatmaps of the COVID-19 versus normal pCXR are shown in Figure 4. The CNN algorithm was able to localize the area of pathology on pCXR. For CNN performed on the whole $\mathrm{pCXR}$, the majority of the hot spots were reasonably localized to regions 
230 of ground glass opacities and/or consolidations, but some hot spots were located outside the lungs.

231 For CNN performed on segmented lungs, the majority of the hot spots were reasonably localized

232 to regions of ground glass opacities and/or consolidations, mostly as expected. There were a few

233 pixels outside the lung that the algorithm paid attention to. These "errors" could be due to small sample

234 sizes. It learned from the training dataset and there may be information that the algorithm might consider

235 important. Large sample size usually minimizes such "error."

236

237 Discussion

238

This study developed and applied a deep-learning CNN algorithm with transfer learning to

239 classify COVID-19 CXR from normal, bacterial pneumonia, and non-COVID viral pneumonia

240 CXR in a multiclass model. Heatmaps showed reasonable localization of abnormalities in the

241 lungs. The overall sensitivity, specificity, accuracy, and AUC were 0.91, 0.93, 0.88, and .89

242 respectively (segmented lungs).

243

244

There are a few AI studies to date using machine learning methods to classify CXRs of

COVID-19, normal and related lung infections. By the time this paper is reviewed many more

papers will be published. Hurt et al. used a U-net CNN algorithm to predict pixel-wise probability

maps for pneumonia on CXR on 10 COVID-19 patients (Hurt et al. 2020). No ROC analysis was

performed. Apostolopoulos and Mpesiana used deep-learning algorithm to predict COVID-19 
254 cohorts were highly asymmetric. Murphy et al. used an artificial intelligence to classify COVID-

25519 CXRs (N=223) from non-COVID-19 CXRs $(\mathrm{N}=231)$ with an 0.81 AUC and they also showed

256 that AI outperformed expert readers (Murphy et al. 2020). Ozturk et al. used an AI model to

257 perform multiclass classification for COVID-19 (N=127) vs. No-Findings (N=500) vs. Pneumonia

$258(\mathrm{~N}=500)$ as well as a binary classification for COVID vs. No-Findings which achieved 87.02\%

259 and 98.08\% accuracies, respectively(Ozturk et al. 2020). Pereira et al. performed a multiclass

260 classification and a hierarchical classification for COVID-19 vs pneumonia vs no-finding using

261 resampling algorithms, texture descriptors, and CNN. This model achieved a F1-Score of 0.65 for

262 the multiclass approach and F1 score of 0.89 for the hierarchical classification (Pereira et al. 2020).

263 AUC and accuracy were not reported. AI has also been employed to stage pCXR disease severity

264 against radiologist scores (Cohen et al. 2020a; Zhu et al. 2020a). Our study had one of the larger

265 cohorts, balanced sample sizes, and multi-class model. Our approach is also amongst the simplest

266 AI models with comparable performance index, likely facilitate immediate clinical translation.

267 Together, these studies indicate that AI has the potential to assist frontline physicians in

268 distinguishing COVID-19 infection based on CXRs.

Heatmaps are informative tools to visualize regions that $\mathrm{CNN}$ algorithm pays attention to

271 for detection. This is particular important given AI operates on high dimensional space. Such

272 heatmaps enable reality checks and make AI interpretable with respect to clinical findings. Our

273 algorithm showed that the majority of the hotspots were highly localized to abnormalities within

274 the lungs, i.e., ground glass opacity and/or consolidation, albeit imperfect. The majority of the

275 above-mentioned machine learning studies to classify COVID-19 CXRs did not provide heatmaps.

276 We also noted that CNN on whole pCXR image resulted in some hot spots located outside the 
277 lungs. CNN of segmented lungs solved this problem. Another advantage of using segmented lung

278 is reduced computational cost during training. Transfer learning also reduced computational cost,

279 making this algorithm practical. The performance is generally better using segmented lungs.

280

281 Most COVID-19 positive patients showed significant abnormalities on pCXR. Some early

282 studies have even suggested that pCXR could be used as a primary tool for COVID-19 screening

283 in epidemic areas (Ai et al. 2020), which could complement swab testing which still has long

284 turnaround time and non-significant false positive rate. In some cases, imaging revealed chest

285 abnormalities even before swab tests confirm infection (Fang et al. 2020, in press; Li et al. 2020a).

286 In addition, $\mathrm{pCXR}$ can detect superimposed bacteria pneumonia, which necessitates urgent

287 antibiotic treatment. pCXR can also suggest acute respiratory distress syndrome, which is

288 associated with severe negative outcomes and necessitates immediate treatment. Together with the

289 potential widespread shortage of intensive care units and mechanical ventilators in many hospitals,

290 pCXR may play a critical role in decision-making. A timely implementation of AI methods could

291 help to realize the full potential of pCXR in this COVID-19 pandemic.

292

293

This pilot proof-of-principal study has several limitations. This is a retrospective study with

294

a small sample size and the data sets used for training had limited alternative diagnoses. Although

295 the Kaggle database has a large sample size for non-COVID-19 CXR, we chose the sample sizes

296 to be comparable to that of COVID-19 to avoid asymmetric sample sizes that could skew

297 sensitivity and specificity. Future studies will need to increase the COVID-19 sample size and

298 include additional lung pathologies. The spatiotemporal characteristics on pCXR of COVID-19

299 infection and its relation to clinical outcomes are unknown. Future endeavors could include 
300 developing AI algorithms to stage severity, and predict progression, treatment response,

301 recurrence, and survival, to inform and advise risk management and resource allocation associated

302 with the COVID-19 pandemic, with inclusion of clinical variables in predictive models (Lam et

303 al. 2020; Zhao et al. 2020; Zhu et al. 2020b).

305 In conclusion, deep learning convolutional neural networks with transfer learning 306 accurately classify COVID-19 pCXR from pCXR of normal, bacterial pneumonia, and non307 COVID viral pneumonia patients in a multi-class neural nwork model. This approach has the 308 potential to help radiologists and frontline physicians by providing efficient and accurate 309 diagnosis.

310

311

312 


\section{REFERENCES}

314

315 https://coronavirus.jhu.edu/map.html. Johns Hopkin University.

316 Ai T, Yang Z, Hou H, Zhan C, Chen C, Lv W, Tao Q, Sun Z, and Xia L. 2020. Correlation of

317 Chest CT and RT-PCR Testing in Coronavirus Disease 2019 (COVID-19) in China: A

$318 \quad$ Report of 1014 Cases. Radiology 296:E32-E40.

319 Apostolopoulos ID, and Mpesiana TA. 2020. Covid-19: automatic detection from X-ray images

320

321

322

323

324

325

326

327

328

329

330

331

332

333

334

335

336

337

338

339

340

341

342

343

344

345

346

347

348

349

350

351

352

353

354

355

356 640. 10.1007/s13246-020-00865-4

Bao C, Liu X, Zhang H, Li Y, and Liu J. 2020. Coronavirus Disease 2019 (COVID-19) CT Findings: A Systematic Review and Meta-analysis. J Am Coll Radiol 17:701-709. 10.1016/j.jacr.2020.03.006

Byra M, Styczynski G, Szmigielski C, Kalinowski P, Michalowski L, Paluszkiewicz R, Ziarkiewicz-Wroblewska B, Zieniewicz K, Sobieraj P, and Nowicki A. 2018. Transfer learning with deep convolutional neural network for liver steatosis assessment in ultrasound images. Int J Comput Assist Radiol Surg 13:1895-1903. 10.1007/s11548-018$1843-2$

CDC. https://www.cdc.gov/coronavirus/2019-ncov/lab/index.html.

Cohen JP, Dao L, Roth K, Morrison P, Bengio Y, Abbasi AF, Shen B, Mahsa HK, Ghassemi M, Li H, and Duong TQ. 2020a. Predicting COVID-19 Pneumonia Severity on Chest X-ray With Deep Learning. Cureus 12:e9448. 10.7759/cureus.9448

Cohen JP, Morrison P, and Dao L. 2020b. COVID-19 Image Data Collection. arXiv:200311597v1.

Ehteshami Bejnordi B, Mullooly M, Pfeiffer RM, Fan S, Vacek PM, Weaver DL, Herschorn S, Brinton LA, van Ginneken B, Karssemeijer N, Beck AH, Gierach GL, van der Laak J, and Sherman ME. 2018. Using deep convolutional neural networks to identify and classify tumor-associated stroma in diagnostic breast biopsies. Mod Pathol 31:15021512. 10.1038/s41379-018-0073-z

Elaziz MA, Hosny KM, Salah A, Darwish MM, Lu S, and Sahlol AT. 2020. New machine learning method for image-based diagnosis of COVID-19. PLoS One 15:e0235187. 10.1371/journal.pone.0235187

Fang Y, Zhang H, Xie J, Lin M, Ying L, Pang P, and Ji W. 2020, in press. Sensitivity of Chest CT for COVID-19: Comparison to RT-PCR. Radiology.

Harris M, Qi A, Jeagal L, Torabi N, Menzies D, Korobitsyn A, Pai M, Nathavitharana RR, and Ahmad Khan F. 2019. A systematic review of the diagnostic accuracy of artificial intelligence-based computer programs to analyze chest x-rays for pulmonary tuberculosis. PLoS One 14:e0221339. 10.1371/journal.pone.0221339

Heo SJ, Kim Y, Yun S, Lim SS, Kim J, Nam CM, Park EC, Jung I, and Yoon JH. 2019. Deep Learning Algorithms with Demographic Information Help to Detect Tuberculosis in Chest Radiographs in Annual Workers' Health Examination Data. Int J Environ Res Public Health 16. 10.3390/ijerph16020250

Holshue ML, DeBolt C, Lindquist S, Lofy KH, Wiesman J, Bruce H, Spitters C, Ericson K, Wilkerson S, Tural A, Diaz G, Cohn A, Fox L, Patel A, Gerber SI, Kim L, Tong S, Lu X, Lindstrom S, Pallansch MA, Weldon WC, Biggs HM, Uyeki TM, Pillai SK, and 
Washington State -nCo VCIT. 2020. First Case of 2019 Novel Coronavirus in the United States. N Engl J Med 382:929-936. 10.1056/NEJMoa2001191

Hui DS, E IA, Madani TA, Ntoumi F, Kock R, Dar O, Ippolito G, McHugh TD, Memish ZA, Drosten C, Zumla A, and Petersen E. 2020. The continuing 2019-nCoV epidemic threat of novel coronaviruses to global health - The latest 2019 novel coronavirus outbreak in Wuhan, China. Int J Infect Dis 91:264-266. 10.1016/j.ijid.2020.01.009

Hurt B, Kligerman S, and Hsiao A. 2020. Deep Learning Localization of Pneumonia: 2019 Coronavirus (COVID-19) Outbreak. J Thorac Imaging 35:W87-W89. 10.1097/RTI.0000000000000512

Krizhevsky A, Sutskever I, and Hinton GE. 2012. Imagenet classification with deep convolutional neural networks. Advances in neural information processing systems. $\mathrm{p}$ 1097-1105.

Lam KW, Chow KW, Vo J, Hou W, Li H, Richman PS, Mallipattu SK, Skopicki HA, Singer AJ, and Duong TQ. 2020. Continued in-hospital ACE inhibitor and ARB Use in hypertensive COVID-19 patients is associated with positive clinical outcomes. J Infect Dis 222:12561264. 10.1093/infdis/jiaa447

LeCun Y, Bengio Y, and Hinton G. 2015. Deep learning. Nature 521:436-444. 10.1038/nature14539

Li D, Wang D, Dong J, Wang N, Huang H, Xu H, and Xia C. 2020a. False-Negative Results of Real-Time Reverse-Transcriptase Polymerase Chain Reaction for Severe Acute Respiratory Syndrome Coronavirus 2: Role of Deep-Learning-Based CT Diagnosis and Insights from Two Cases. Korean J Radiol 21:505-508. 10.3348/kjr.2020.0146

Li K, Wu J, Wu F, Guo D, Chen L, Fang Z, and Li C. 2020b. The Clinical and Chest CT Features Associated With Severe and Critical COVID-19 Pneumonia. Invest Radiol 55:327-331. 10.1097/RLI.0000000000000672

Li Q, Guan X, Wu P, Wang X, Zhou L, Tong Y, Ren R, Leung KSM, Lau EHY, Wong JY, Xing X, Xiang N, Wu Y, Li C, Chen Q, Li D, Liu T, Zhao J, Liu M, Tu W, Chen C, Jin L, Yang R, Wang Q, Zhou S, Wang R, Liu H, Luo Y, Liu Y, Shao G, Li H, Tao Z, Yang Y, Deng Z, Liu B, Ma Z, Zhang Y, Shi G, Lam TTY, Wu JT, Gao GF, Cowling BJ, Yang B, Leung GM, and Feng Z. 2020c. Early Transmission Dynamics in Wuhan, China, of Novel Coronavirus-Infected Pneumonia. N Engl J Med 382:1199-1207. 10.1056/NEJMoa2001316

Lu H, Stratton CW, and Tang YW. 2020. Outbreak of pneumonia of unknown etiology in Wuhan, China: The mystery and the miracle. J Med Virol 92:401-402. 10.1002/jmv.25678

Mekov E, Miravitlles M, and Petkov R. 2020. Artificial intelligence and machine learning in respiratory medicine. Expert Rev Respir Med 14:559-564. 10.1080/17476348.2020.1743181

Murphy K, Smits H, Knoops AJG, Korst M, Samson T, Scholten ET, Schalekamp S, SchaeferProkop CM, Philipsen R, Meijers A, Melendez J, van Ginneken B, and Rutten M. 2020. COVID-19 on the Chest Radiograph: A Multi-Reader Evaluation of an AI System. Radiology:201874. 10.1148/radiol.2020201874

Ozturk T, Talo M, Yildirim EA, Baloglu UB, Yildirim O, and Rajendra Acharya U. 2020. Automated detection of COVID-19 cases using deep neural networks with X-ray images. Comput Biol Med 121:103792. 10.1016/j.compbiomed.2020.103792 
402

403

404

405

406

407

408

409

410

411

412

413

414

415

416

417

418

419

420

421

422

423

424

425

426

427

428

429

430

431

432

433

434

435

436

Pereira RM, Bertolini D, Teixeira LO, Silla CN, Jr., and Costa YMG. 2020. COVID-19 identification in chest X-ray images on flat and hierarchical classification scenarios. Comput Methods Programs Biomed 194:105532. 10.1016/j.cmpb.2020.105532

Ren T, Cattell R, Duanmu H, Huang P, Li H, Vanguri R, Liu MZ, Jambawalikar S, Ha R, Wang F, Cohen J, Bernstein C, Bangiyev L, and Duong TQ. 2020. Convolutional Neural Network Detection of Axillary Lymph Node Metastasis Using Standard Clinical Breast MRI. Clin Breast Cancer 20:e301-e308. 10.1016/j.clbc.2019.11.009

Samala RK, Chan HP, Hadjiiski LM, Helvie MA, Cha KH, and Richter CD. 2017. Multi-task transfer learning deep convolutional neural network: application to computer-aided diagnosis of breast cancer on mammograms. Phys Med Biol 62:8894-8908. 10.1088/1361-6560/aa93d4

Simonyan K, and A. Z. 2014. Very Deep Convolutional Networks for Large-Scale Image Recognition. arXiv:14091556.

Song J, Hu B, Qu H, Wang L, Huang X, Li M, and Zhang M. 2020. Upregulation of angiotensin converting enzyme 2 by shear stress reduced inflammation and proliferation in vascular endothelial cells. Biochem Biophys Res Commun 525:812-818.

10.1016/j.bbrc.2020.02.151

Tang YW, Schmitz JE, Persing DH, and Stratton CW. 2020. Laboratory Diagnosis of COVID19: Current Issues and Challenges. J Clin Microbiol 58. 10.1128/JCM.00512-20

Wang W, Xu Y, Gao R, Lu R, Han K, Wu G, and Tan W. 2020. Detection of SARS-CoV-2 in Different Types of Clinical Specimens. JAMA 323:1843-1844. 10.1001/jama.2020.3786

Xu X, Yu C, Qu J, Zhang L, Jiang S, Huang D, Chen B, Zhang Z, Guan W, Ling Z, Jiang R, Hu T, Ding Y, Lin L, Gan Q, Luo L, Tang X, and Liu J. 2020. Imaging and clinical features of patients with 2019 novel coronavirus SARS-CoV-2. Eur J Nucl Med Mol Imaging 47:1275-1280. 10.1007/s00259-020-04735-9

Zhao Z, Chen A, Hou W, Graham JM, Li H, Richman PS, Thode HC, Singer AJ, and Duong TQ. 2020. Prediction model and risk scores of ICU admission and mortality in COVID-19. PLoS One 15:e236618. 10.1371/journal.pone.0236618

Zhu J, Shen B, Abbasi A, Hoshmand-Kochi M, Li H, and Duong TQ. 2020a. Deep transfer learning artificial intelligence accurately stages COVID-19 lung disease severity on portable chest radiographs. PLoS One 15:e0236621. 10.1371/journal.pone.0236621

Zhu JS, Ge P, Jiang C, Zhang Y, Li X, Zhao Z, Zhang L, and Duong TQ. 2020b. Deep-learning artificial intelligence analysis of clinical variables predicts mortality in COVID-19 patients. J Am Coll Emerg Physicians Open. 10.1002/emp2.12205 


\section{Figure legends}

440

441 Figure 1. VGG16 architecture with 16 weighted layers including 3 fully connected layers.

443 Figure 2: Examples of chest radiographs (a) normal, (b) COVID-19 viral pneumonia, (c) non444 COVID-19 viral pneumonia, and (d) bacterial pneumonia. COVID-19 is often characterized by 445 ground-glass opacities with or without nodular consolidation with predominance of bilateral, 446 peripheral and lower lobes involvement. Non-COVID-19 viral pneumonia is often characterized 447 by diffuse interstitial opacities, usually bilaterally. Bacterial pneumonia is often characterized by 448 confluent areas of focal airspace consolidation. Arrows indicate regions of above-described 449 characteristic features.

450

451 Figure 3: CNN (a) training and (b) validation loss and accuracy. Loss decreases and accuracy 452 improved with increasing epoch for both training and validation dataset.

453

454 Figure 4: (a) pCXR from a COVID-19 patient, (b) the corresponding segmented lung, (c) heatmap 455 from $\mathrm{CNN}$ analysis using whole $\mathrm{pCXR}$, and (d) heatmap from $\mathrm{CNN}$ analysis using segmented lung 456 overlaid on whole CXR. Arrows indicated regions of ground glass opacity and/or consolidations. 457 


\section{Table 1 (on next page)}

Confusion table

Table 1. Confusion table showing the multiclass CNN classification (whole CXR) 
Table 1. Confusion table showing the multiclass CNN classification (whole CXR)

\begin{tabular}{|c|c|c|c|c|}
\hline & Normal & $\begin{array}{c}\text { COVID- } \\
\mathbf{1 9}\end{array}$ & $\begin{array}{c}\text { Non-COVID- } \\
\mathbf{1 9} \text { viral } \\
\text { pneumonia }\end{array}$ & $\begin{array}{c}\text { Bacterial } \\
\text { pneumonia }\end{array}$ \\
\hline Normal & 122 & 3 & 17 & 2 \\
\hline Covid19 & 6 & 102 & 3 & 6 \\
\hline $\begin{array}{c}\text { Non-COVID-19 viral } \\
\text { Pneumonia }\end{array}$ & 16 & 2 & 94 & 20 \\
\hline Bacterial pneumonia & 4 & 1 & 30 & 85 \\
\hline
\end{tabular}

2 
Table 2 (on next page)

Precision and recall rate and F1 score (whole CXR).

Table 2 shows the precision and recall rate and F1 score (whole CXR). 
1 Table 2 shows the precision and recall rate and F1 score (whole CXR).

\begin{tabular}{|c|c|c|c|}
\hline & Precision & Recall & F1 - score \\
\hline Normal & 0.82 & 0.85 & 0.84 \\
\hline Covid19 & 0.94 & 0.87 & 0.91 \\
\hline $\begin{array}{c}\text { Non-covid19 viral } \\
\text { pneumonia }\end{array}$ & 0.65 & 0.71 & 0.68 \\
\hline Bacterial pneumonia & 0.75 & 0.71 & 0.73 \\
\hline
\end{tabular}

2 
Figure 1

\section{VGG16 architecture}

Figure 1. VGG16 architecture with 16 weighted layers including 3 fully connected layers. 
Input

\begin{tabular}{|c|}
\hline Conv2D 1-1 \\
\hline Conv2D 1-2 \\
\hline Pooling \\
\hline
\end{tabular}

Conv2D 2-1

Conv2D 2-2

\begin{tabular}{|c|}
\hline Pooling \\
\hline Conv2D 3-1 \\
\hline Conv2D 3-2 \\
\hline Conv2D 3-3 \\
\hline Pooling \\
\hline Conv2D 4-1 \\
\hline Conv2D 4-2 \\
\hline Conv2D 4-3 \\
\hline
\end{tabular}

Pooling

Conv2D 5-1

Conv2D 5-2

Conv2D 5-3

Pooling

\begin{tabular}{|l|}
\hline Dense \\
\hline Dense \\
\hline Dense \\
\hline
\end{tabular}

Output 


\section{Figure 2}

CXR

Figure 2: Examples of chest radiographs (a) normal, (b) COVID-19 viral pneumonia, (c) nonCOVID-19 viral pneumonia, and (d) bacterial pneumonia. COVID-19 is often characterized by ground-glass opacities with or without nodular consolidation with predominance of bilateral, peripheral and lower lobes involvement. Non-COVID-19 viral pneumonia is often characterized by diffuse interstitial opacities, usually bilaterally. Bacterial pneumonia is often characterized by confluent areas of focal airspace consolidation. Arrows indicate regions of above-described characteristic features. 


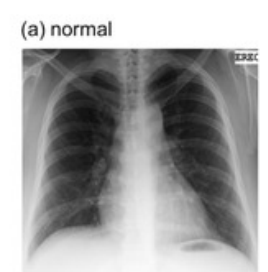

(b) COVID-19 pneumonia

(c) non-COVID-19 viral pneumonia

(d) bacterial pneumonia
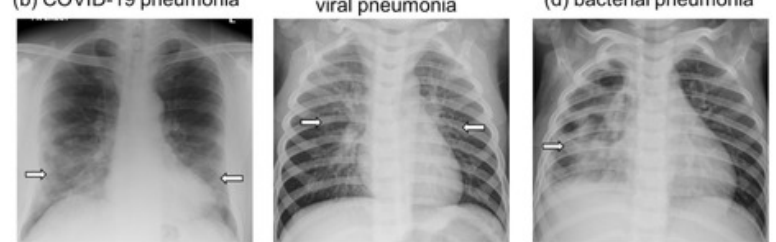
Figure 3

CNN training and validation

Figure 3: CNN training and validation loss and accuracy. Loss decreases and accuracy improved with increasing epoch for both training and validation dataset. 

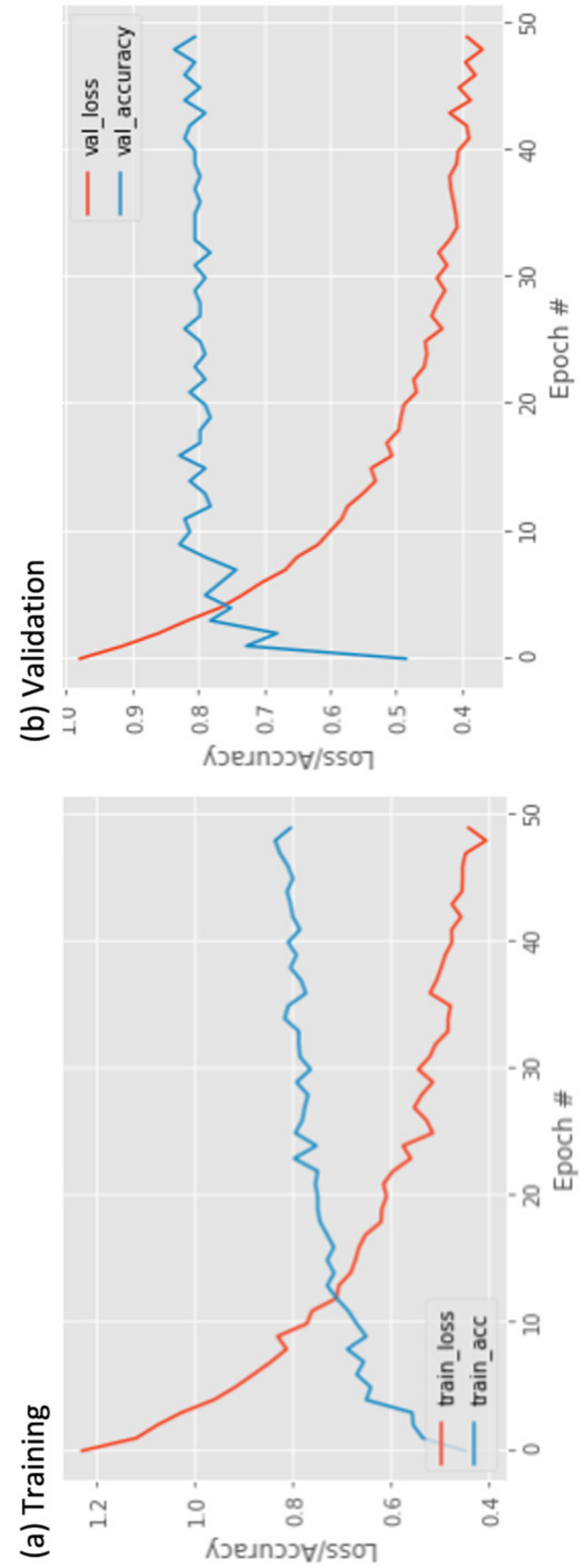
Figure 4

Heatmap

Figure 4: pCXR from a COVID-19 patient, the corresponding segmented lung, heatmap from CNN analysis using whole $\mathrm{pCXR}$, and heatmap from CNN analysis using segmented lung overlaid on whole CXR. Arrows indicated regions of ground glass opacity and/or consolidations. 

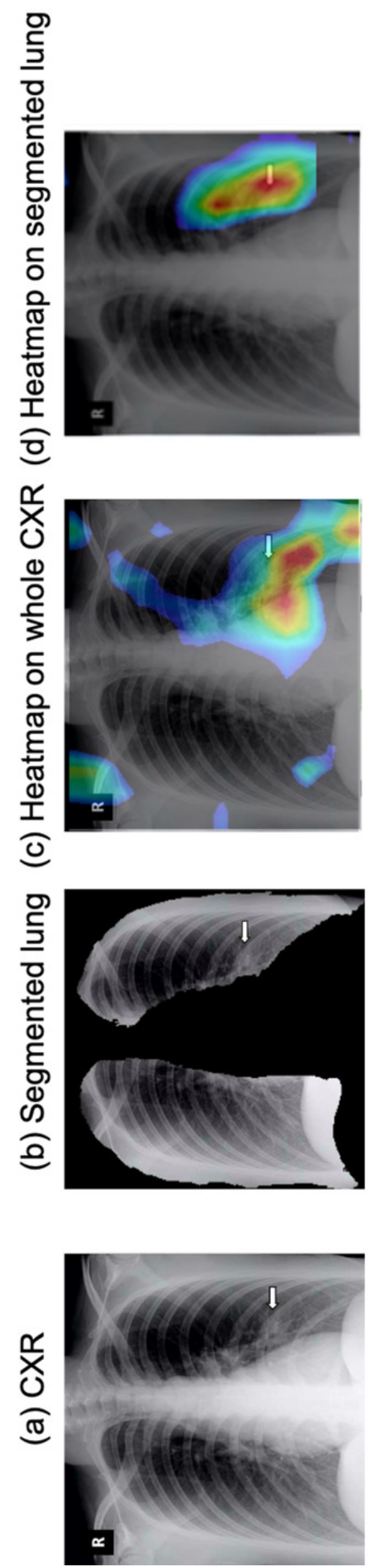